\title{
Impact diurnal variations of IOP on dynamic corneal hysteresis measured with air-puff swept-source OCT
}

\author{
Karol Karnowski, ${ }^{* 1,2,3}$ Ewa Mączyńska, ${ }^{1}$ Maciej Nowakowski, ${ }^{1}$ Bartłomiej Kałużny, ${ }^{4}$ Ireneusz Grulkowski ${ }^{1}$ and \\ Maciej Wojtkowski ${ }^{1,2}$ \\ ${ }^{1}$ Institute of Physics, Nicolaus Copernicus University, ul. Grudziądzka 5, 87-100 Toruń, Poland, \\ ${ }^{2}$ Institute of Physical Chemistry, Polish Academy of Sciences, ul. Kasprzaka44/52, Warsaw, Poland 44/52, \\ Warsaw, Poland \\ ${ }^{3}$ School of Electrical, Electronic and Computer Engineering, The University of Western Australia, 35 Stirling \\ Highway, Perth, Western Australia 6009, Australia \\ ${ }^{4}$ Division of Ophthalmology and Optometry, Department of Ophthalmology, Collegium Medicum, Nicolaus \\ Copernicus University, Bydgoszcz, Poland
}

Received September 11, 2018; accepted September 26, 2018; published September 30, 2018

\begin{abstract}
The deformation amplitudes measured with air-puff OCT are sensitive to both (intraocular pressure) IOP and biomechanical properties of the cornea. Amplitudes analysis of corneal deformations is challenging due to interrelation of IOP and corneal biomechanics. In this study, we used natural diurnal IOP fluctuations to investigate corneal deformations in a number of subjects whose eyes were measured multiple times during the day. The results of analysis, based on corneal hysteresis, revealed a corneal hysteresis parameter, which remains constant during the day for each individual eye. We hypothesize that the above-mentioned metric might correlate with biomechanical properties of the cornea without any influence of IOP.
\end{abstract}

The structures of the anterior segment of the human eye play a crucial role in our ability to clearly perceive the visual world that surrounds us. Besides the structural information, one can measure other quantities of the anterior segment of the human eye. Two of them intraocular pressure (IOP) and biomechanical properties of the cornea - play a vital role not only in some anterior segment pathologies (e.g. keratoconus [1-3]) but can also trigger retina related pathologies (e.g. glaucoma [4-6]).

A range of different tonometers are used in clinical practice to measure the IOP. A standard procedure determines the IOP by detecting the force that is required to flatten the cornea over some specified area. Either mechanical probe (applanation tonometry) or rapid air pulse (air-puff tonometry) can be used. The latter approach is a key in non-contact tonometry, which is considered to be less invasive. An electro-optic detection system measures the force of an applied air stream enabling IOPestimation. A similar system allows for measurements of temporal corneal deformations induced by an air pulse recorded as changes in the intensity of light reflected from the cornea. Indirectly measured deformations provide additional information on corneal biomechanics. It must be noticed here that estimated IOP

\footnotetext{
${ }^{*}$ E-mail: a.author@if.pw.edu.pl
}

and biomechanical properties are sensitive to individual eye parameters such as central corneal thickness (CCT) [7-9] and radius of curvature [10].

In 2011, we presented a novel method and instrument for direct measurements of dynamic corneal deformation during air puff [11]. The Swept Source Optical Coherence Tomography (SSOCT) system combined with a custom adapted air puff chamber, due to its unique parameters, allows tracking the dynamics of not only corneal surfaces but also the posterior surface of the crystalline lens. The system was designed in such a way that an OCT beam was collinear with an air stream applied on the cornea. It was shown that corneal deformation amplitude was sensitive to IOP changes. However, no correlation with a commercial device (Tono-Pen) was found.

Later on, another group demonstrated a similar approach but the Spectral OCT system was used to image the cornea [12]. In that study, two separate systems $-800 \mathrm{~nm}$ OCT and commercial air-puff tonometer - were combined with the use of a tilted mirror attached to the tonometer exit pipe. The system allowed the measurements of a single point corneal apex as well as full cross-sectional monitoring of corneal deformation. It was demonstrated that corneal deformation amplitude is sensitive to biomechanical properties of the cornea (before and after collagen cross-linking procedure on the animal model eye).

An alternative commercial approach (OCULUS Corvis ${ }^{\circledR}$ ST), which was introduced contemporaneously to OCT tonometry, is based on a Scheimpflug imaging method well-known from a clinically proven corneal topographer - OCULUS PENTACAM. The device records airinduced deformations of the cornea with a high-speed camera, with a rate of 4300 cross-sectional images per second. However, the advantage of OCT imaging is that it provides a better quality of images compared to the Scheimpflug camera [13] 
In this paper, we present a hysteresis curve analysis applied to air-puff SSOCT measurements of corneal deformations. The procedures do not require any changes of the previously used SSOCT system [11]. The analysis of corneal deformations for 10 healthy eyes, measured at different time points during a day is presented.

The system used in this study (Fig. 1) was described in details elsewhere [11]. SSOCT, which we used previously for $3 \mathrm{D}$ imaging of the anterior segment of the human eye [13], was combined with the air-puff chamber adapted from a commercial tonometer (XPert NCT; Reichert Inc.). A wavelength-tunable laser (Axsun Technologies), operating at $1310 \mathrm{~nm}$ central wavelength with a $100 \mathrm{~nm}$ bandwidth and a $50 \mathrm{kHz}$ repetition rate, was used as a light source. The system provides a $9 \mu \mathrm{m}$ axial resolution and an imaging depth of $9 \mathrm{~mm}$.

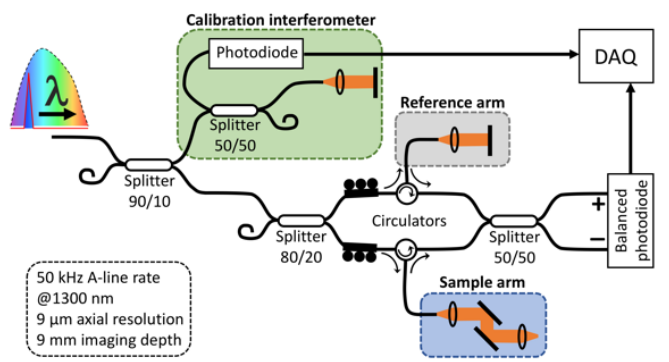

Fig. 1. Schematic of the swept source OCT system used for the study.

The cornea was illuminated with $2.5 \mathrm{~mW}$, well below the safety power levels given by the ANSI standard [14]. The imaging head of the system was combined and aligned with air-puff chamber to guarantee collinear operation of an OCT beam and air stream applied to the cornea. A single air-puff pulse was triggered by the analog output of a PCI-7631 board (National Instruments) and synchronized with a pair of galvanometric scanners and data acquisition. The system directly acquires a time series of A-scans at a single point on the cornea (M-scan). Before every measurement session, reference interferometric fringes (common path interferometer) were recorded with the second channel of a digitizer (GaGe CompuScope 14200, DynamicSignals LLC). This step was necessary to remap an OCT signal to the wavenumber domain before FFT is performed. During the measurements of corneal deformation, the second channel was used to record the signal from the pressure sensor installed in the air-puff chamber. This signal carries temporal information representing the strength of an air stream applied to deform the cornea.

In our previous work [11], we showed that corneal deformation amplitude is sensitive to IOP (changes in intraocular pressure were introduced with Alphagan drops - an IOP-reducing agent). The influence of corneal biomechanics on deformation amplitudes was investigated in a separate test when the corneal apex and the peripheral part were measured consecutively in a time interval short enough to assume no significant change of IOP. Lower deformation amplitudes were observed for peripheral measurements (Fig. 2). However, we cannot draw any conclusions from two measurements only, but the observation seems to be in agreement with the previous findings by Dorronsoro at.al. on animal models [12]. The influence of corneal thickness on deformation amplitudes has been so far unexplored for air-puff OCT measurements. We may suspect that for two cases where only corneal thickness was changed (IOP, biomechanical properties of the cornea remains constant) lower deformation amplitude would be observed for a thicker cornea.

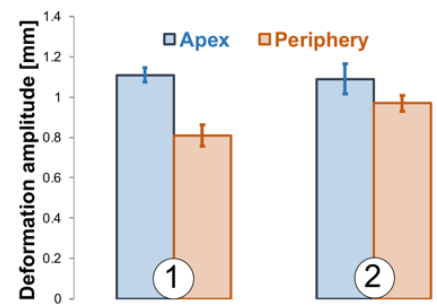

Fig. 2. Differences in deformation amplitude measured in corneal apex and at peripheries. Corneal thickness for each condition is given in corresponding graph columns. The numbers in circles denote eyes used in the experiment. Error bars are \pm SEM error $\left(\mathrm{SEM}=\mathrm{SD} /\left(\mathrm{n}^{1 / 2}\right)\right.$, where $n$ $=3$ is the number of measurements for each case).

Since the amplitude of air-induced corneal deformation is sensitive to IOP, corneal biomechanics and potentially to corneal thickness, we decided to perform additional experiments that rely on natural IOP fluctuations during the day [15]. Approval from the university human research ethics committee was obtained prior to commencement of these studies. Before the measurements, all healthy volunteers were examined by a trained clinician to ensure no ocular pathologies.

Ten eyes of 5 healthy volunteers were measured in eight sessions during the day (100-minute interval between sessions). Each eye was first measured three times with a handheld applanation tonometer Tono-Pen (Reichert Inc., Buffalo, NY), followed by three air-puff SSOCT measurements. The participants were also asked to spend time between the sessions in accordance with their typical daily habits. From each OCT measurement, a corneal deformation was extracted by automatic segmentation procedures (LabView) and amplitudes of deformations were calculated. The obtained results were corrected for the refractive index to obtain geometric distances. Additionally, the temporal profile of pressure generated in air-puff chamber was measured with an external pressure sensor.

We compared diurnal fluctuations of deformation amplitude and Tono-Pen IOP readout. The highest observed diurnal IOP change among all measured eyes 
was $\Delta \mathrm{IOP}_{\max }=9 \mathrm{mmHg}, \quad$ while the lowest $\Delta \mathrm{IOP}_{\min }=2 \mathrm{mmHg}$. The intraocular pressure levels for all participants were within the normal IOP range $(10 \mathrm{mmHg}$ to $21 \mathrm{mmHg}$ ) with three exceptions (twice $9.33 \mathrm{mmHg}$ and once $8.67 \mathrm{mmHg}$ ). Similarly, the highest observed diurnal change of deformation amplitude was $\Delta \mathrm{DA}_{\max }=0.23 \mathrm{~mm}$ and the lowest $\Delta \mathrm{DA}_{\min }=0.08 \mathrm{~mm}$. We found the changes of both quantities during the day to be significant, however no correlation between Tono-Pen IOP and deformation amplitude was found.

Straightforward analysis of deformation amplitude cannot provide sufficient information to estimate IOP with high reliability. Therefore, in the approach used here the information on pressure generated in the air-puff chamber (Fig. 3A blue curve) was combined with the deformation of the anterior corneal surface (Fig. 3A red curve).
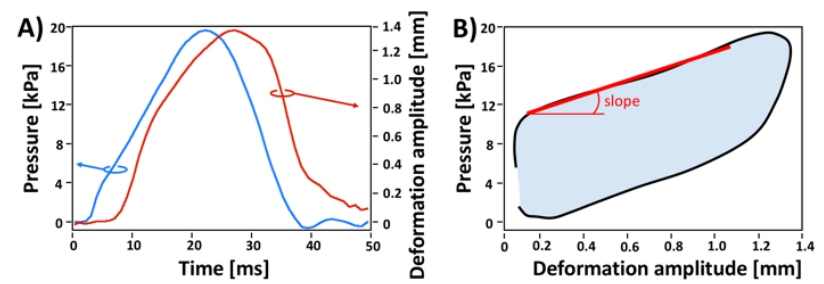

Fig. 3. Hysteresis curve analysis. A) Temporal profiles of applied pressure (blue) and apex deformation of the anterior corneal surface (red). B) Hysteresis curve plotted as deformation amplitude versus applied pressure (plotted from data presented on Fig. 5A). Linear fit (thick red) used to calculate a hysteresis loading slope.

We calibrated the sensor installed in the air-puff chamber with an additional external pressure sensor. We plot the amplitude of deformation versus applied pressure [16] The resulting hysteresis curve (Fig. 3B) is similar to classical mechanics stress-strain plots for viscoelastic materials.

We performed hysteresis curve analysis on our diurnal measurements and we found very interesting regularity (Fig. 4A). If hysteresis curves for individual eye throughout all diurnal sessions are plotted we clearly see that part of the curve (loading cycle) remains unchanged for all of the sessions, while the other part (unloading cycle) differs between the measurements. Mean diurnal hysteresis slopes for the linear section of a loading cycle (Fig. 3B - red line) was calculated for each individual eye (Fig. 4B). The slope extracted from the hysteresis curve remains constant for an individual eye throughout the day, but differs when different eyes from different volunteers are compared.

Assuming than corneal biomechanics remains unchanged during the day, one can hypothesize that the extracted slope of a corneal hysteresis curve might correlate with the biomechanical properties of the cornea without any IOP influence. Confirmation whether this statement is valid requires further studies including animal model ex vivo measurements and Finite Element Method modeling of cornea deformation.

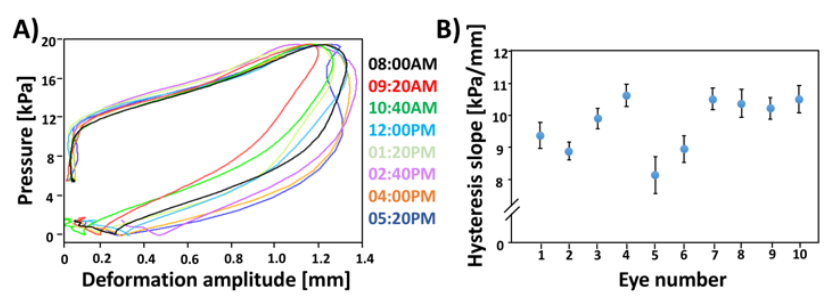

Fig. 4. A) Diurnal hysteresis curves for a randomly chosen eye exposing regularity of a loading cycle of the curves. B) Mean diurnal hysteresis slopes plotted for each individual eye measured.

To conclude, we performed diurnal measurements of airinduced corneal deformations with a custom-build airpuff swept-source OCT system. The measurements were analyzed using hysteresis curves, where both deformation as well as applied force were used. The results of analysis, based on corneal hysteresis, revealed a parameter of the corneal hysteresis curve which remains constant during the day for each individual eye. Interestingly, differences for the same parameter were observed when different eyes were compared.

This project has received funding from the European Union's Horizon 2020 research and innovation programme under grant agreements No 779960 and No 666295 .

\section{References}

[1] K.M. Meek, S.J. Tuft, Y. Huang, P.S. Gill, S. Hayes, R.H. Newton, A.J. Bron, Invest. Ophthalmol. Vis. Sci. 46(6), 1948 (2005).

[2] D.R. Zimmermann, R.W. Fisher, K.H. Winterhalter, R. Witmer, L. Vaughan, Exp. Eye Res. 46(3), 431 (1988).

[3] T.T. Andreassen, A.H. Simonsen, and H. Oxlund, Exp. Eye Res. 31(4), 435 (1980)

[4] A. Heijl, M.C. Leske, B. Bengtsson, L. Hyman, B. Bengtsson, M. Hussein, Arch. Ophthalmol. 120(10), 1268 (2002).

[5] B.C. Chauhan, S.M. Drance, Invest. Ophthal. Visual Sci. 31(11), 2367 (1990).

[6] Y. Gelaw, BMC Ophthalmology 12(1), 58 (2012)

[7] M.J. Doughty, M.L. Zaman, Surv. Ophthalmol. 44(5), 367 (2000).

[8] J. Liu, C.J. Roberts, J. Cataract. Refract. Surg. 31(1), 146 (2005).

[9] N. Ehlers, F.K. Hansen, H. Aasved, Acta Ophthalmol. 53(4), 652 (1975)

[10] Y. Harada, N. Hirose, A. Tawara, J. Glaucom. 17(8), 619 (2008).

[11] D. Alonso-Caneiro, K. Karnowski, B.J. Kaluzny, A. Kowalczyk, M. Wojtkowski, Opt. Expr. 19(15), 14188 (2011).

[12] C. Dorronsoro, D. Pascual, P. Perez-Merino, S. Kling, S. Marcos, Biomed. Opt. Expr. 3(3), 473 (2012).

[13] K. Karnowski, B.J. Kaluzny, M. Szkulmowski, M. Gora, M. Wojtkowski, Biomed. Opt. Expr. 2(9), 2709 (2011).

[14] American National Standard for Safe use of Lasers (American National Standards Institute, Orlando, FL, 2000)

[15] R. David, L. Zangwill, D. Briscoe, M. Dagan, R. Yagev, Y. Yassur, Br. J. Ophthamlom. 76(5), 280 (1992).

[16] E. Maczynska, K. Karnowski, K. Szulzycki, M. Malinowska, H. Dolezyczek, A. Cichanski, M. Wojtkowski, B.J. Kaluzny, I. Grulkowski, J. Biophoton. (to be published) 